\title{
COMPUTER-ADAPTIVE BALANCE TESTING IMPROVES DISCRIMINATION BETWEEN COMMUNITY-DWELLING ELDERLY FALLERS AND NON-FALLERS
}

\author{
Poonam K. Pardasaney, ScD ${ }^{1,2, *}$, Pengsheng Ni, MD², Mary D. Slavin, PhD², Nancy K. \\ Latham, $\mathbf{P h D}^{2}$, Robert C. Wagenaar, $\mathrm{PhD}^{3,4,{ }^{* *}}$, Jonathan Bean, $\mathbf{M D}^{5}$, and Alan M. Jette, $\mathbf{P h D}^{2}$
}

${ }^{1}$ Department of Rehabilitation Sciences, Sargent College of Health \& Rehabilitation Sciences, Boston University ${ }^{2}$ Health \& Disability Research Institute, School of Public Health, Boston University ${ }^{3}$ Department of Physical Therapy and Athletic Training, Sargent College of Health \& Rehabilitation Sciences, Boston University ${ }^{4}$ Rudolf Magnus Institute of Neuroscience \& Department of Rehabilitation, Nursing Sciences and Sports, University Medical Center Utrecht, Utrecht, the Netherlands ${ }^{5}$ Department of Physical Medicine and Rehabilitation, Spaulding Rehabilitation Hospital Network, Harvard Medical School

\begin{abstract}
Objective-To build an item response theory based computer-adaptive balance test (CAT) from three traditional, fixed-form balance measures: Berg Balance Scale (BBS), Performance-Oriented Mobility Assessment (POMA), and Dynamic Gait Index (DGI); and examine whether CAT psychometric performance exceeded that of individual measures.

Design-Secondary analysis combining two existing datasets.

Setting-Community-based.

Participants-187 community-dwelling older adults, 65 years or older, mean age $75.2 \pm 6.8$ years, $69 \%$ female.

Interventions-Not applicable.

Main Outcome Measure(s)_BBS, POMA, and DGI items were compiled into an initial 38item bank. Rasch Partial Credit Model was used for final item bank calibration. CAT simulations were conducted to identify the ideal CAT. CAT score accuracy, reliability, floor and ceiling
\end{abstract}

(C) 2014 The American Congress of Rehabilitation Medicine. Published by Elsevier Inc. All rights reserved.

Corresponding author: Poonam K. Pardasaney, ScD RTI International, 1440 Main Street, Suite 337, Waltham, MA 02451 poonamp@ @u.edu; pardasaney@ rti.org Business: 781434 1711; Mobile: 8579284349.

RTI International, 1440 Main Street, Suite 337, Waltham, MA 02451

** Dr. Robert C. Wagenaar passed away on February 13, 2013, prior to manuscript submission.

Publisher's Disclaimer: This is a PDF file of an unedited manuscript that has been accepted for publication. As a service to our customers we are providing this early version of the manuscript. The manuscript will undergo copyediting, typesetting, and review of the resulting proof before it is published in its final citable form. Please note that during the production process errors may be discovered which could affect the content, and all legal disclaimers that apply to the journal pertain.

Reprint requests to Dr. Poonam K. Pardasaney. 
effects, and validity were examined. Floor and ceiling effects and validity of CAT and individual measures were compared.

Results-A 23-item bank met model expectations. A 10-item CAT was selected, showing very strong association with full item bank scores ( $r=0.97)$, and good overall reliability $(0.78)$.

Reliability was better in low- to mid-balance ranges due to better item targeting to balance ability, compared with highest balance ranges. No floor effect was noted. CAT ceiling effect (11.2\%) was significantly lower than POMA (40.1\%) and DGI $(40.3 \%)$ ceiling effects $(p<0.0001$ per comparison). The CAT outperformed individual measures, being the only test to discriminate between fallers and non-fallers ( $p=0.0068)$, and strongest predictor of self-reported function.

Conclusions-The balance CAT showed excellent accuracy, good overall reliability, and excellent validity compared with individual measures, being the only measure to discriminate between fallers and non-fallers. Prospective examination, particularly in low- functioning elderly and clinical populations with balance deficits, is recommended. Development of an improved CAT based on an expanded item bank containing higher difficulty items is also recommended.

\section{Keywords}

computer-adaptive testing; postural balance; aged

Performance-based balance assessment is fundamental in geriatric rehabilitation, given the negative impact of balance impairment on function and fall risk. ${ }^{1}$ While used extensively to assess balance and monitor change, existing balance measures have important limitations in community-dwelling elderly, including limited comprehensiveness in content, ceiling effects, and limited sensitivity to change and responsiveness. ${ }^{2-5}$ These limitations likely contribute to multiple test administration for assessment, ${ }^{6,7}$ increasing burden. In clinical trials, measures with these limitations can lead to type II errors and falsely diminished effect sizes. ${ }^{2}$ In clinical settings, these limitations render measures susceptible to reduced diagnostic sensitivity and reduced ability to capture improvement from intervention, particularly in individuals with mild deficits.

Most balance measures are traditional fixed-form measures, requiring fixed item set administration to every person. Designing psychometrically strong fixed-form balance measures is challenging due to the large number of items needed to encompass the spectrum of balance ability and components. ${ }^{8}$ Other authors acknowledge that the multifactorial nature of balance precludes development of all-encompassing measures, ${ }^{9}$ and combinations of measures must be used for assessment. ${ }^{10}$ Additionally, depending on an individual's balance ability and dysfunction, comprehensive assessment may not be needed. Computeradaptive testing, a contemporary method based on item response theory (IRT),${ }^{8}$ offers a promising approach for overcoming limitations of fixed-form balance measures and designing comprehensive, tailored, efficient, and responsive tests. IRT models describe the association between individual's ability level and probability of a particular item response, providing item-based scores rather than test-based scores and allowing score comparison when individuals have not completed identical items. ${ }^{8,11}$ CAT programs use simple artificial intelligence that selects the optimal items from a calibrated IRT-item bank based on individual ability level, avoiding inappropriately easy or difficult items ${ }^{8}$ and adjusts test 
length to achieve desired precision. Being tailored to individuals' ability level, CATs can provide precise scores while reducing administration time associated with fixed-form tests. While CATs have been extensively applied to patient-reported outcomes assessment, ${ }^{8,12-15}$ their application to performance-based assessments remains new.

A balance CAT developed for the stroke population showed promising psychometric properties and reduced testing time compared with the fixed-form Berg Balance Scale (BBS). ${ }^{16}$ The item bank, however, excluded walking and stair climbing, limiting applicability to ambulatory, community-dwelling elderly. To our knowledge, a balance CAT for community-dwelling elderly has not been reported.

The purpose of this study was to develop and examine psychometric properties of a balance CAT for community-dwelling elderly based on three fixed-form measures: BBS, ${ }^{17,18}$ Performance-Oriented Mobility Assessment (POMA), ${ }^{4,19}$ and Dynamic Gait Index (DGI). ${ }^{20,21}$ The study had three aims: (i) to create an IRT-based item bank combining BBS, POMA, and DGI items; (ii) to build a CAT from the item bank and examine its score accuracy, reliability, floor and ceiling effects, and known-groups and predictive validity; and (iii) to compare floor and ceiling effects and validity of the CAT against individual balance measures. We hypothesized that the CAT would provide superior validity over individual measures because of greater item bank comprehensiveness, and testing tailored to individuals' balance ability.

\section{METHODS}

The study, involving secondary analysis, was approved by Institutional Review Boards of Boston University and Spaulding Rehabilitation Hospital, Boston, MA.

\section{Sample}

Data from two studies of ambulatory community-dwelling elderly, 65 years or older, conducted in outpatient rehabilitation facilities in Greater Boston were combined. The first was a 16-week randomized controlled trial comparing two exercise interventions in 138 volunteer subjects, ${ }^{22}$ baseline BBS, POMA, and DGI scores, and 16-week Late-Life Function and Disability Instrument-Function Scale (LLFDI-Function) scores were used. The second study cross-sectionally examined the relationship between balance and falls in 49 volunteer subjects; ${ }^{23} \mathrm{BBS}$, POMA, and DGI scores were used. The first study required independence climbing a flight of stairs, while the second evenly recruited fallers and nonfallers. Exclusion criteria were neuromuscular impairment limiting participation; ${ }^{22}$ unstable disease ${ }^{22}$ specific medical diagnoses or findings, such as orthostatic hypotension, that could affect balance or fall status; ${ }^{23}$ and cognitive impairment. ${ }^{22,23}$ The first study defined fallers as 'persons reporting any mechanical fall within the past year', and the second as 'persons reporting two or more mechanical falls within the past year'.

\section{Balance Measures}

BBS, POMA, and DGI items were compiled into an initial 38-item bank, higher scores indicating better balance., 17-21 The 14-item BBS primarily assesses transfers and standing balance on a 0-4 scale. ${ }^{17,18}$ POMA contains a 9-item balance subscale (PB) assessing 
transfers and standing balance, and 7-item gait subscale (PG) comprising observational gait analysis, both rated on a $0-1$ or $0-2$ scale. ${ }^{4,19}$ The 8 -item DGI assesses balance during gait on a $0-3$ scale. ${ }^{20,21}$

\section{Self-Reported Function Measure}

LLFDI-Function, ${ }^{24}$ used for predictive validity testing, contains 32 items assessing upper and lower extremity function on a 1-5 scale. ${ }^{24}$ Raw scores are converted to scaled summary scores from 0-100, higher scores indicating better function. LLFDI-Function has shown high test-retest reliability (intraclass correlation coefficient $=0.77-0.98$ ) in community-dwelling elderly, and known-groups validity in distinguishing among persons with varying levels of functional limitation or cane use. ${ }^{24,25}$ LLFDI-Function scores show moderate to strong associations with balance, ${ }^{25,26}$ and were available on 115 subjects from the first sub-sample.

\section{Item Bank Development}

Rasch Partial Credit Model, a one-parameter IRT model that estimates item difficulty, was applied for item bank development using Winteps, ${ }^{27,28}$ as outlined below.

a. Rating Scale Analysis: Each balance item rating scale was evaluated using three criteria:

(i) $\geq 10$ observations per category; (ii) increase in average item measures with increasing category difficulty; and (iii) category outlier-sensitive (outfit) mean square (MnSq) values $<2.0$, to eliminate unexplained noise. ${ }^{29}$ Items were deleted when rating scales failed to meet these criteria despite category collapsing. The highest rating scale category (e.g., BBS category 4) was never combined with lower categories.

b. Unidimensionality: Unidimensionality, indicating items in a scale measure a single construct (e.g., balance), ${ }^{30,31}$ was established via three methods. First, one-factor confirmatory factor analysis (CFA) was conducted using MPlus (Muthén \& Muthén, 2010, Los Angeles, CA), with comparative fit index (CFI) and Tucker-Lewis Index (TLI) $\searrow 0.95$, and root mean square error of approximation (RMSEA) $<0.06$ indicating unidimensionality. ${ }^{31} \mathrm{CFA}$ factor loadings, reflecting strength of association between an item and overall factor, were examined with loadings $>0.4$ being desirable. ${ }^{32}$ Second, principal components analysis (PCA) of the standardized Rasch residual matrix was conducted, with first residual component eigenvalue $<3$ supporting unidimensionality. ${ }^{30,33}$ Third, items were dichotomized into subsets loading positively or negatively on the first Rasch residual component and subject scores from item subsets were compared using paired $t$-tests, unidimensionality supported by non-significance at the 0.05 level. ${ }^{30}$

c. Local Independence: Local independence states that persons' responses are determined only by ability level, and once underlying ability is controlled, item responses are uncorrelated. ${ }^{31}$ Local independence was assessed using Rasch inter-item residual correlation matrix, correlations $>0.7$ indicating highly locally-dependent pairs. ${ }^{30}$ One item from each locally-dependent pair was deleted, the preference to delete items showing lower item fit and difficulty (below); redundant content; involvement in further locally-dependent pairs; and lower point-measure correlation coefficients, representing association between item observations and person scores. ${ }^{30}$ 
d. Item Fit: Item fit, reflecting agreement between observed and expected item responses, ${ }^{33}$ was assessed via information-weighted (infit) $\mathrm{MnSq}$, sensitive to inlying data patterns, and outfit MnSq, sensitive to outliers. While we ensured acceptable outfit MnSq, we only deleted items with infit $\mathrm{MnSq}>1.5$, as large infit $\mathrm{MnSq}$ poses a greater validity threat than large outfit MnSq. 30,34

e. Differential Item Functioning (DIF): Items functioned differentially if difficulty calibrations differed across subgroups with equal balance ability levels. ${ }^{31}$ DIF analysis was conducted across the following subgroups: (i) younger vs. older groups, using median age as cutpoint; (ii) gender; (iii) fallers vs. non-fallers; and (iv) the two study sub-samples. Two methods were used: (i) DIF analysis in Winsteps using independent $t$-tests, and (ii) ordinal logistic regression using Statistical Analysis Software, Version 9.1 (SAS Institute Inc., SAS Campus Drive, Cary, NC). For independent $t$-tests, Holm's Sequential Bonferroni correction was applied to maintain a 0.05 family-wise error rate across item comparisons for each grouping variable, test significance indicating item DIF. Two ordinal logistic regression models were run per item; the dependent variable was raw item score; model 1 independent variable, raw total score; and model 2 independent variables, raw total score and grouping variable. A $10 \%$ change in slope estimate of raw total score from model 1 to model 2 indicated DIF. ${ }^{35}$ Items showing DIF by both methods were handled as follows: (i) separate subgroup calibrations for age, gender, or falling status DIF items; or (ii) elimination of sample DIF items, as separate sample calibrations would be irrelevant for prospective application. ${ }^{31}$

f. Item Calibration, Balance Ability, Item Targeting: In the final step, Partial Credit Model was applied to calibrate item difficulty parameters for each item in log-odds units (logits). Average item difficulty was fixed at zero logits, higher scores indicating greater difficulty. Subjects' balance ability logit scores were transformed to a standardized scale with mean 50 and S.D. 10, higher scores indicating better balance. ${ }^{36}$ To assess whether items were welltargeted to sample balance ability, the range of item difficulty estimates was plotted against that of subjects' ability estimates. ${ }^{30}$

\section{CAT Simulations}

Using the final item bank, CAT algorithms were created using HDR software (HDR, Boston University, MA). CAT simulations were conducted, in which actual responses to administered items were entered from the dataset. A common first item of mid-difficulty was administered in all simulations. At each subsequent step, weighted maximum likelihood estimated person scores and S.E.s based on previous responses. Following each response, the item providing maximum information function at the provisional score level was administered, information function referring to amount of information provided about person ability. ${ }^{37}$ These steps were repeated until all final bank items had been administered, to allow comparison of CATs of varying lengths.

Score accuracy and conditional reliability of CATs of varying lengths were compared for final CAT selection. Score accuracy, defined as strength of association between CAT and full item bank scores, was assessed using Pearson correlation coefficients. Conditional reliability was examined to determine whether reliability varied with balance ability, using 
the formula $1 /\left[1+(\text { standard error })^{2}\right],{ }^{38}$ values $\searrow 0.7$ being acceptable. ${ }^{15}$ Overall CAT reliability was calculated as $\left.1 /\left[1+\{\text { mean*(standard error })^{2}\right\}\right] .{ }^{39}$ Floor and ceiling effects were calculated as percentage of persons scoring only in lowest and highest rating scale categories, respectively. Item exposure rate was calculated as ratio of total number of times an item was administered against total number of CAT administrations, equaling 187.

\section{CAT Validity Testing}

Using analysis of covariance (ANCOVA) to control for study sub-sample, known-groups validity was examined by assessing ability of CAT scores to discriminate between fallers and non-fallers. To examine predictive validity, multiple linear regression with a 0.05 significance level was used to assess ability of CAT scores to predict 16-week LLFDIFunction scores, while controlling for age, gender, body mass index, trial intervention group, and number of medical diagnoses.

\section{Comparison of CAT and Individual Balance Measures}

Floor and ceiling effects of CAT and individual measures were compared using McNemar's test for dependent data and 0.05 significance level. Known-groups validity of individual measures was examined as above, and compared with CAT results; to maintain 0.05 familywise error rate across the four ANCOVA comparisons, a 0.0125 significance level was used. Predictive validity of CAT and individual measures was compared via significance of slope estimates and Type II partial $\mathrm{R}^{2}$, larger partial $\mathrm{R}^{2}$ indicating stronger prediction.

\section{RESULTS}

Sample characteristics are presented in Table 1. Mean balance scores indicated mild impairment on these tests; mean LLFDI-Function scores indicated mild functional limitations. Ten items were deleted from the initial 38-item bank because of $<10$ observations in the lowest rating scale category, despite collapsing to dichotomous scales (Supplemental E-Table). Twenty rating scales were collapsed because of unobserved categories or $<10$ observations per category; one was further collapsed due to poor category fit (i.e., category outfit MnSq >2.0). Finally, 26 items had a dichotomous scale; one, a 3point scale; and one, a 5-point scale.

The 28-item bank did not satisfy unidimensionality (CFI=0.91;TLI=0.91; RMSEA=0.076; first Rasch residual component eigenvalue=3.2). However, Rasch inter-item residual correlation matrix revealed three locally-dependent item pairs with correlation coefficients of 0.67, 0.64, and 0.52. After deletion of one item from each pair (Supplemental E-Table), the remaining 25 -item bank demonstrated unidimensionality (CFI=0.96;TLI=0.96; RMSEA=0.047; first residual component eigenvalue $=2.8$ ). Further, there was no significant difference in subjects' balance ability scores from item subsets loading positively and negatively on the first residual component (mean difference=-0.1;S.D. $=1.7 ; p=0.43$ ). No age, gender, or falling status DIF was noted. However, two items showing sample DIF were deleted, including BBS7 'standing with feet together' (DIF contrast $=2.26$ logits; $p=0.0003$ ) and DGI8 'negotiating stairs' (DIF contrast $=1.55$ logits; $p=0.0009$ ). The final 23 -item bank showed unidimensionality $(\mathrm{CFI}=0.97 ; \mathrm{TLI}=0.96 ; \mathrm{RMSEA}=0.048$; first residual component 
eigenvalue=2.7), local independence (largest inter-item residual correlation coefficient=0.37), and good item fit (infit $\mathrm{MnSq}=0.68-1.37$; outfit $\mathrm{MnSq}=0.37-1.49$ ), with CFA factor loadings from 0.49 to 0.95 (Table 2).

Item calibration is shown in Table 2, the most difficult item being BBS14 'standing on one leg', and easiest, PB3 'attempts to arise'. Distribution of item category difficulty and balance ability scores revealed good item targeting to balance ability in low- to mid-balance ranges; however, there were few higher difficulty items to target persons of highest balance abilities (Fig. 1).

The first item in CAT simulations was PB9 'sitting down'. After examining score accuracy and conditional reliability (Supplemental E-Fig. 1) of CATs of various lengths, a 10-item CAT was selected. In terms of accuracy, 10-item CAT scores were very strongly associated with full item bank scores $(r=0.97)$. All CATs provided poor reliability in highest balance ranges, with no noticeable improvement with full item bank administration (Supplemental E-Fig. 1). 10-item CAT provided above acceptable reliability across the remaining balance continuum, all estimates exceeding 0.75 in the 23-54 score range. 5-item CAT reliability was below acceptable in lowest balance ranges (Supplemental E-Fig. 1). For the 10-item CAT, $89 \%$ of reliability estimates exceeded 0.7 ; for the full item bank, $91 \%$ exceeded 0.7 . While a longer CAT improved reliability in lower balance ranges, a 10-item CAT was selected for efficiency. Overall 10-item CAT reliability was 0.78 .

No floor effects were noted. Ceiling effects on 10-item CAT were 11.2\%; BBS, 14.4\%; POMA, 40.1\%; and DGI, 40.3\%. In comparison, full item bank ceiling effect was $8.6 \%$. CAT ceiling effects were significantly lower than POMA $(p<0.0001)$ and DGI $(p<0.0001)$ ceiling effects, while CAT and BBS ceiling effects were not significantly different $(p=0.08)$. The 10 most difficult items had the highest item exposure rates (Table 2, Supplemental EFig. 2).

Known-groups validity testing revealed the CAT was the only measure that significantly discriminated between fallers and non-fallers; BBS, POMA, and DGI were not significant discriminators (Table 3). While baseline CAT and individual balance measure scores significantly predicted 16-week self-reported function scores, comparison of partial $\mathrm{R}^{2}$ values revealed the CAT was the strongest predictor (Table 4).

\section{DISCUSSION}

Our study demonstrates that a 10-item balance CAT developed from fixed-form measures showed superior validity compared with the fixed-form measures in community-dwelling elderly. The CAT was the only test that discriminated fallers from non-fallers, and was a stronger predictor of self-reported function compared with individual measures. A key CAT strength was its' ability to combine existing measures into a single measure with superior validity, without increasing testing burden. The 10 most commonly-administered CAT items represented an even distribution of BBS, PB, and DGI items, demonstrating that collective items from different measures, rather than items from any single measure, provided maximum information about balance ability. The 10 most commonly-administered CAT 
items also represented the most difficult items, likely improving ability to discriminate fall status.

Our finding of low item targeting to balance ability in highest balance ranges was not unexpected as BBS, POMA, and DGI have known ceiling effects in community-dwelling elderly. ${ }^{2,3}$ CAT reliability was better in low- to mid-balance ranges, where improved item targeting to balance ability reduced standard error of assessment. Despite reduced item targeting and reliability in highest balance ranges, superior validity of the CAT compared with individual measures is very promising.

Similar to our findings, the stroke balance CAT developed by Hsueh et al. ${ }^{16}$ had higher reliability in persons with moderate balance scores than in those with extreme scores. Compared with our CAT, the stroke balance CAT had better reliability and item targeting to sample ability, likely because its items were specifically developed for a stroke population and calibrated in a large patient sample with a wide range of balance abilities. Additionally, compared with our use of a one-parameter IRT model, use of a two-parameter model to calibrate the stroke CAT item bank likely contributed to higher reliability. ${ }^{36}$

Given the prevalence of balance problems in older adults ${ }^{40}$ and morbidity and mortality associated with falls, ${ }^{41}$ a psychometrically strong and efficient balance measure that is a more sensitive indicator of fall risk would have important applications in geriatric rehabilitation. The promising performance of our CAT supports its prospective examination, particularly in low-functioning elderly and clinical populations with balance deficits. Further, our findings strongly support development of an improved balance CAT based on an expanded item bank that fills gaps in item difficulty noted in our study. Recent research by our group provides important insights into content areas that could be incorporated to develop higher difficulty balance items. ${ }^{5}$

\section{Study Limitations}

Being a secondary analysis, our study has some limitations. First, several items were deleted or modified due to inadequate lower rating scale category frequencies, partly due to our high-functioning sample. Majority of rating scales were dichotomous scales, which have lower information function than polytomous scales. Nevertheless, Rasch analyses of BBS and DGI scores in lower-functioning elderly samples undergoing balance rehabilitation also revealed unobserved or underutilized rating scale categories. ${ }^{42,43,44}$ Second, our sample size precluded two-parameter IRT model application, which may have improved score reliability. Nonetheless, Rasch models offer practical advantages, including parsimony and simplicity of interpretation based on total scores alone. ${ }^{36,37}$ Third, two items were deleted because of sample DIF. While DGI8 DIF is likely explained by the independent stair climbing requirement in the 138 -subject sample, ${ }^{22}$ we could not explain BBS7 DIF. Although DIF can be accidental and non-replicable, ${ }^{45}$ we deleted DIF items as separate sample calibrations would be irrelevant for future application. Fourth, our use of the same data for CAT development and validation may have biased our results. Prospective CAT validation in different samples is recommended to further support its validity. Finally, while our item bank was limited to BBS, POMA, and DGI items, incorporation of useful items from other measures is recommended in future item bank expansion. 


\section{CONCLUSIONS}

A balance CAT for community-dwelling elderly developed from three fixed-form measures showed excellent accuracy, good overall reliability, and superior validity compared with individual measures, the CAT being the only measure that discriminated between fallers and non-fallers. Prospective CAT examination, particularly in low-functioning elderly and clinical populations with balance deficits, is recommended. Development of an improved balance CAT based on an expanded item bank containing higher difficulty items is also recommended.

\section{Supplementary Material}

Refer to Web version on PubMed Central for supplementary material.

\section{Acknowledgments}

Dr. Pardasaney was supported by the Dudley Allen Sargent Research Fund from Sargent College of Health \& Rehabilitation Sciences, Boston University. Drs. Slavin and Jette were supported by an R24 award (1R24HD065688-02) from the National Institutes of Health -National Center for Medical Rehabilitation Research. Dr. Bean was supported by K23 (K23AG019663-01A2) and K24 (K24HD070966-02) awards from the National Institutes of Health.

\section{LIST OF ABBREVIATIONS}

$\begin{array}{ll}\text { ANCOVA } & \text { Analysis of Covariance } \\ \text { BBS } & \text { Berg Balance Scale } \\ \text { CAT } & \text { Computer Adaptive Test(ing) } \\ \text { CFA } & \text { Confirmatory Factor Analysis } \\ \text { CFI } & \text { Comparative Fit Index } \\ \text { DGI } & \text { Dynamic Gait Index } \\ \text { DIF } & \text { Differential Item Functioning } \\ \text { Infit } & \text { MnSq Information-Weighted Mean Square } \\ \text { IRT } & \text { Item Response Theory } \\ \text { LLFDI-Function } & \text { Late-Life Function and Disability Instrument - Function Scale } \\ \text { Outfit } & \text { MnSq Outlier-Sensitive Mean Square } \\ \text { PB } & \text { Performance-Oriented Mobility Assessment - Balance Subscale } \\ \text { PCA } & \text { Principal Components Analysis } \\ \text { PG } & \text { Performance-Oriented Mobility Assessment - Gait Subscale } \\ \text { POMA } & \text { Performance-Oriented Mobility Assessment } \\ \text { RMSEA } & \text { Root Mean Square Error of Approximation } \\ \text { TLI } & \text { Tucker-Lewis Index }\end{array}$




\section{REFERENCES}

1. Muir SW, Berg K, Chesworth B, Klar N, Speechley M. Quantifying the magnitude of risk for balance impairment on falls in community-dwelling older adults: A systematic review and metaanalysis. J Clin Epidemiol. 2010; 63(4):389-406. [PubMed: 19744824]

2. Pardasaney PK, Latham NK, Jette AM, et al. Sensitivity to change and responsiveness of four balance measures for community-dwelling older adults. Phys Ther. 2012; 92(388-397)

3. Lundin-Olsson L. Community-dwelling older adults with balance impairment show a moderate increase in fall risk, although further research is required to refine how balance measurement can be used in clinical practice. Evid Based Nurs. 2010; 13(3):96-97. doi: 10.1136/ebn.13.3.96. [PubMed: 20584842]

4. Shumway-Cook, A.; Woollacott, M. Motor Control: Translating Research into Clinical Practice. 3rd ed.. Lippincott Williams \& Wilkins; Philadelphia, PA: 2007. Clinical management of the patient with a postural control disorder.; p. 257-295.

5. Pardasaney PK, Slavin MD, Wagenaar RC, Latham NK, Ni P, Jette AM. Conceptual limitations of balance measures for community-dwelling older adults. Phys Ther. 2013; 93(10):1351-1368. doi: 10.2522/ptj.20130028; 10.2522/ptj.20130028. [PubMed: 23704036]

6. Sibley KM, Straus SE, Inness EL, Salbach NM, Jaglal SB. Balance assessment practices and use of standardized balance measures among Ontario physical therapists. Phys Ther. 2011; 91(11):15831591. [PubMed: 21868613]

7. Beling J, Roller M. Multifactorial intervention with balance training as a core component among fall-prone older adults. J Geriatr Phys Ther. 2009; 32(3):125-133. [PubMed: 20128337]

8. Jette AM, Haley SM. Contemporary measurement techniques for rehabilitation outcomes assessment. J Rehabil Med. 2005; 37(6):339-345. doi: 10.1080/16501970500302793. [PubMed: 16287664]

9. Dite W, Temple VA. A clinical test of stepping and change of direction to identify multiple falling older adults. Arch Phys Med Rehabil. 2002; 83(11):1566-1571. [PubMed: 12422327]

10. Berg K, Norman KE. Functional assessment of balance and gait. Clin Geriatr Med. 1996; 12(4): 705-723. [PubMed: 8890112]

11. Hambleton RK. Emergence of item response modeling in instrument development and data analysis. Med Care. 2000; 38(9):II60-II65. Supplement II: Health Outcomes Methodology: Symposium Proceedings. [PubMed: 10982090]

12. Haley SM, Siebens H, Coster WJ, et al. Computerized adaptive testing for follow-up after discharge from inpatient rehabilitation: I. Activity outcomes. Arch Phys Med Rehabil. 2006; 87(8):1033-1042. doi: 10.1016/j.apmr.2006.04.020. [PubMed: 16876547]

13. Cella D, Riley W, Stone A, et al. The patient-reported outcomes measurement information system (PROMIS) developed and tested its first wave of adult self-reported health outcome item banks: 2005-2008. J Clin Epidemiol. 2010; 63(11):1179-1194. [PubMed: 20685078]

14. Cella D, Yount S, Rothrock N, et al. The patient-reported outcomes measurement information system (PROMIS): Progress of an NIH roadmap cooperative group during its first two years. Med Care. 2007; 45(5 Suppl 1):S3-S11. [PubMed: 17443116]

15. McDonough CM, Tian F, Ni P, et al. Development of the computer-adaptive version of the late-life function and disability instrument. J Gerontol A Biol Sci Med Sci. 2012 doi: 10.1093/gerona/ gls 108.

16. Hsueh IP, Chen JH, Wang CH, et al. Development of a computerized adaptive test for assessing balance function in patients with stroke. Phys Ther. 2010; 90(9):1336-1344. [PubMed: 20592269]

17. Berg K, Wood-Dauphinee SL, Williams JI, Gayton D. Measuring balance in the elderly: Preliminary development of an instrument. Physiotherapy Canada. 1989; 41(6):304-310.

18. Berg KO, Wood-Dauphinee SL, Williams JI, Maki B. Measuring balance in the elderly: Validation of an instrument. Can J Public Health. Jul-Aug;1992 83(Suppl 2):S7-11. [PubMed: 1468055]

19. Tinetti ME. Performance-oriented assessment of mobility problems in elderly patients. J Am Geriatr Soc. 1986; 34(2):119-126. [PubMed: 3944402]

20. Shumway-Cook A, Baldwin M, Polissar NL, Gruber W. Predicting the probability for falls in community-dwelling older adults. Phys Ther. 1997; 77(8):812-819. [PubMed: 9256869] 
21. Shumway-Cook, A.; Woollacott, M. Motor Control: Translating Research into Clinical Practice. 3rd ed.. Lippincott Williams \& Wilkins; Philadelphia, PA: 2007. Clinical management of the patient with a mobility disorder.; p. 390-440.

22. Bean JF, Kiely DK, LaRose S, O'Neill E, Goldstein R, Frontera WR. Increased velocity exercise specific to task training versus the National Institute on Aging's strength training program: Changes in limb power and mobility. J Gerontol A Biol Sci Med Sci. 2009; 64(9):983-991. doi: 10.1093/gerona/glp056. [PubMed: 19414509]

23. Slavin, MD.; Lipsitz, LA.; Bean, J.; Phillips, E. Development of calibrated test items for measuring balance performance in community-dwelling elderly individuals.. Gerontologist; Abstracts of the 55th annual meeting of the Gerontological Society of America; Boston, Massachusetts, USA. 22-26 November 2002; 2002. p. 1-435.Spec 1

24. Haley SM, Jette AM, Coster WJ, et al. Late life function and disability instrument: II. Development and evaluation of the function component. J Gerontol A Biol Sci Med Sci. 2002; 57(4):M217-22. [PubMed: 11909886]

25. Melzer I, Kurz I, Sarid O, Jette AM. Relationship between self-reported function and disability and balance performance measures in the elderly. J Rehabil Res Dev. 2007; 44(5):685-691. [PubMed: 17943680]

26. Melzer I, Kurz I. Self reported function and disability in late life: A comparison between recurrent fallers and non-fallers. Disabil Rehabil. 2009; 31(10):791-798. doi: 10.1080/09638280802309384. [PubMed: 19034779]

27. Bond, TG.; Fox, CM. The Partial Credit Rasch model.. In: Bond, TG.; Fox, CM., editors. Applying the Rasch Model: Fundamental Measurement in the Human Sciences. 2nd ed.. Lawrence Erlbaum Associates; Mahwah, NJ: 2007. p. 123-142.

28. Linacre, JM. Winsteps ${ }^{\circledR}$ Rasch measurement computer program. 2012.

29. Linacre JM. Optimizing rating scale category effectiveness. J Appl Meas. 2002; 3(1):85-106. [PubMed: 11997586]

30. Linacre, JM. Winsteps $®$ Rasch Measurement Computer Program User's Guide. Beaverton, Oregon: 2012.

31. Reeve BB, Hays RD, Bjorner JB, et al. Psychometric evaluation and calibration of health-related quality of life item banks: Plans for the patient-reported outcomes measurement information system (PROMIS). Med Care. 2007; 45(5 Suppl 1):S22-31. [PubMed: 17443115]

32. Hair, JF.; Anderson, RE.; Tatham, RL.; Black, W. Multivariate Data Analysis. Prentice-Hall; New Delhi: 1998.

33. Bond, TG.; Fox, CM. Model fit and unidimensionality.. In: Bond, TG.; Fox, CM., editors. Applying the Rasch Model: Fundamental Measurement in the Human Sciences. 2nd ed.. Lawrence Erlbaum Associates; Mahwah, NJ: 2007. p. 253

34. Wright BD, Linacre JM. Reasonable mean-square fit values. Rasch Measurement Transactions. 1994; 8(3):370.

35. Crane PK, Gibbons LE, Jolley L, van Belle G. Differential item functioning analysis with ordinal logistic regression techniques. DIFdetect and difwithpar. Med Care. 2006; 44(11 Suppl 3):S11523. [PubMed: 17060818]

36. Bjorner JB, Chang CH, Thissen D, Reeve BB. Developing tailored instruments: Item banking and computerized adaptive assessment. Qual Life Res. 2007; 16(Suppl 1):95-108. [PubMed: 17530450]

37. Thomas ML. The value of item response theory in clinical assessment: A review. Assessment. 2011; 18(3):291-307. [PubMed: 20644081]

38. Masse LC, Heesch KC, Eason KE, Wilson M. Evaluating the properties of a stage-specific selfefficacy scale for physical activity using classical test theory, confirmatory factor analysis and item response modeling. Health Educ Res. 2006; 21(Suppl 1):33-46.

39. Cheng Y, Yuan K, Liu C. Comparison of reliability measures under factor analysis and item response theory. Educational and Psychological Measurement. 2012; 72(1):52-67. doi: $10.1177 / 0013164411407315$.

40. Dillon CF, Gu Q, Hoffman HJ, Ko CW. Vision, hearing, balance, and sensory impairment in Americans aged 70 years and over: United states, 1999-2006. NCHS data brief. 2010; (31) 
41. Rubenstein LZ. Falls in older people: Epidemiology, risk factors and strategies for prevention. Age Ageing. 2006; 35(Suppl 2):37-41. [PubMed: 16364932]

42. Kornetti DL, Fritz SL, Chiu YP, Light KE, Velozo CA. Rating scale analysis of the Berg Balance Scale. Arch Phys Med Rehabil. 2004; 85(7):1128-1135. [PubMed: 15241763]

43. Porta, La; Fabio; Caselli, S.; Susassi, S.; Cavallini, P.; Tennant, A.; Franceschini, M. Is the Berg Balance Scale an internally valid and reliable measure of balance across different etiologies in neurorehabilitation? A revisited Rasch analysis study. Arch Phys Med Rehabil. 2012; 93(7):12091216. doi: 10.1016/j.apmr.2012.02.020. [PubMed: 22521926]

44. Chiu YP, Fritz SL, Light KE, Velozo CA. Use of item response analysis to investigate measurement properties and clinical validity of data for the Dynamic Gait Index. Phys Ther. 2006; 86(6):778-787. [PubMed: 16737403]

45. Du Y. When to adjust for differential item functioning. Rasch Measurement Transactions. 1995; 9(1):414. 


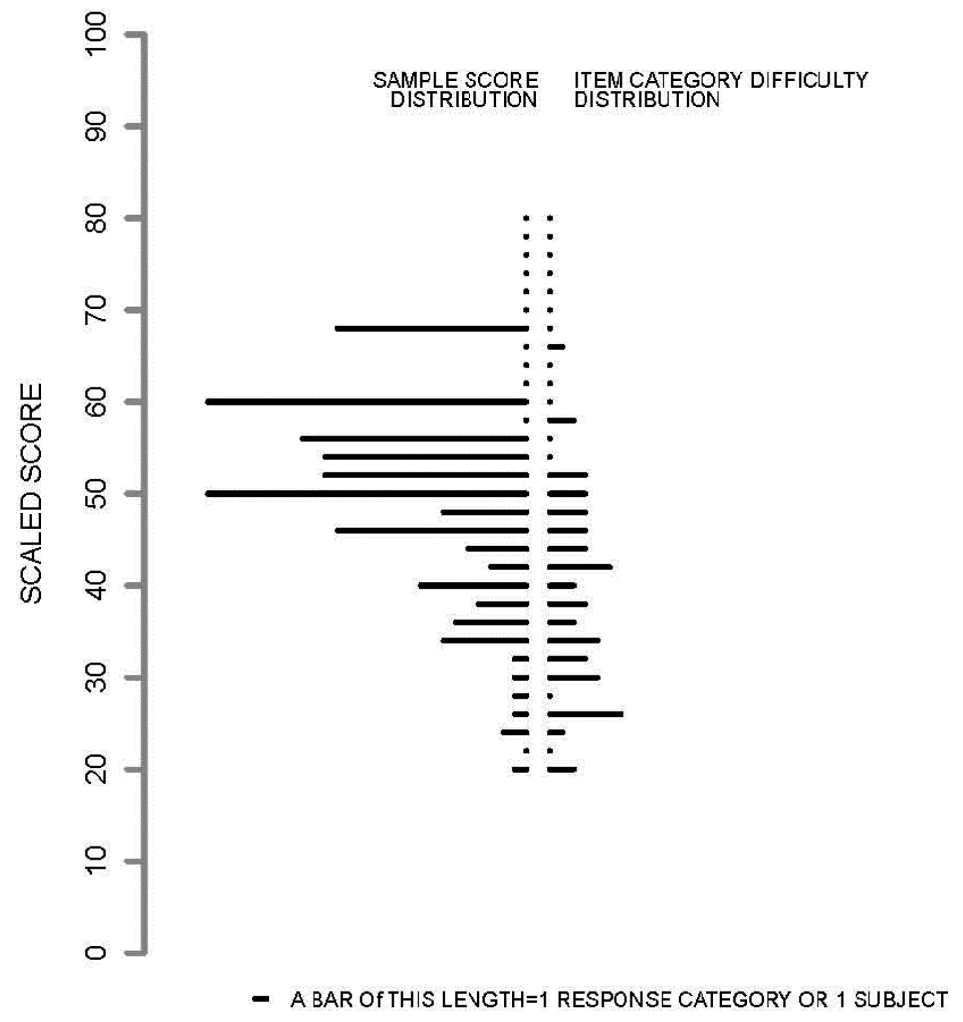

Figure 1.

Distribution of Item Category Difficulty versus Sample Balance Ability. The range of item category difficulty scores is displayed on the right. The range of sample balance ability scores is displayed on the left, where mean $=50$ and S.D. $=10$. 


\section{Table 1}

Descriptive Characteristics

\begin{tabular}{lccc}
\hline & Sample 1 & Sample 2 & Combined Sample \\
\hline Demographic Characteristics & $\boldsymbol{n}=\mathbf{1 3 8}$ & $\boldsymbol{n}=\mathbf{4 9}$ & $\boldsymbol{n}=\mathbf{1 8 7}$ \\
Age in years & $75.4 \pm 6.9$ & $74.8 \pm 6.5$ & $75.2 \pm 6.8$ \\
Age in years (min - max) & $65-94$ & $65-89$ & $65-94$ \\
Female (\%) & 68.8 & 70.0 & 69.0 \\
Fallers (\%) & 24.0 & 47.0 & 30.5 \\
Balance Scores & $\boldsymbol{n}=\mathbf{1 3 8}$ & $\boldsymbol{n}=\mathbf{4 9}$ & $\boldsymbol{n}=\mathbf{1 8 7}$ \\
BBS (0-56) & $50.6 \pm 4.7$ & $52.6 \pm 4.4$ & $51.1 \pm 4.7$ \\
POMA (0-28) & $25.6 \pm 3.0$ & $27.5 \pm 0.7$ & $26.1 \pm 2.7$ \\
DGI (0-24) & $21.3 \pm 3.4$ & $22.2 \pm 2.3$ & $21.5 \pm 3.2$ \\
Self-Reported Physical Function & $\boldsymbol{n}=\mathbf{1 1 5}$ & & \\
LLFDI-Function (0-100) & $65.8 \pm 11.6$ & N/A & N/A \\
\hline
\end{tabular}

Values shown are mean \pm S.D. unless otherwise specified. N/A = not applicable. 
Table 2

Final 23-Item Bank Parameters Displayed in Descending Item Difficulty Order

\begin{tabular}{|c|c|c|c|c|c|c|c|}
\hline Item & Measure $^{+}$(S.E.) & Infit MnSq & Outfit MnSq & PTMEA & CFA Loading & Exposure Rate & Rating Scale ${ }^{*}$ \\
\hline $\begin{array}{l}\text { BBS14: Standing on } \\
\text { one leg }\end{array}$ & $2.05(0.13)$ & 1.25 & 1.25 & 0.61 & 0.56 & 0.89 & 00112 \\
\hline $\begin{array}{l}\text { DGI6: Stepping over } \\
\text { obstacle }\end{array}$ & $1.75(0.18)$ & 0.87 & 0.75 & 0.63 & 0.86 & 0.80 & 0001 \\
\hline BBS13: Tandem stance & $1.46(0.08)$ & 1.37 & 1.30 & 0.74 & 0.66 & 0.93 & 01234 \\
\hline PB6: Nudge & $0.93(0.19)$ & 1.17 & 1.21 & 0.47 & 0.52 & 0.86 & 001 \\
\hline $\begin{array}{l}\text { DGI2: Changing gait } \\
\text { speed }\end{array}$ & $0.66(0.20)$ & 0.77 & 0.62 & 0.65 & 0.89 & 0.88 & 0001 \\
\hline PB2: Arises & $0.61(0.20)$ & 1.22 & 1.48 & 0.42 & 0.62 & 0.87 & 01 \\
\hline $\begin{array}{l}\text { BBS10: Look behind } \\
\text { left \& right }\end{array}$ & $0.57(0.20)$ & 1.27 & 1.40 & 0.41 & 0.54 & 0.89 & 00001 \\
\hline $\begin{array}{l}\text { DGI4: Gait vertical } \\
\text { head turns }\end{array}$ & $0.41(0.21)$ & 1.29 & 1.38 & 0.40 & 0.49 & 0.89 & 0001 \\
\hline PB9: Sitting down & $0.27(0.21)$ & 0.80 & 0.60 & 0.62 & 0.89 & 1.00 & 01 \\
\hline $\begin{array}{l}\text { DGI5: Gait with pivot } \\
\text { turn }\end{array}$ & $0.09(0.22)$ & 0.93 & 0.76 & 0.55 & 0.80 & 0.80 & 0001 \\
\hline BBS8: Forward reach & $-0.02(0.22)$ & 1.10 & 1.25 & 0.45 & 0.63 & 0.02 & 00001 \\
\hline PB5: Standing balance & $-0.02(0.22)$ & 0.91 & 0.65 & 0.56 & 0.86 & 0.07 & 01 \\
\hline BBS5: Transfers & $-0.18(0.23)$ & 0.83 & 0.69 & 0.57 & 0.83 & 0.07 & 00001 \\
\hline $\begin{array}{l}\text { DGI7: Stepping around } \\
\text { obstacle }\end{array}$ & $-0.22(0.23)$ & 1.01 & 0.77 & 0.51 & 0.73 & 0.12 & 0001 \\
\hline $\begin{array}{l}\text { DGI1: Gait level } \\
\text { surface }\end{array}$ & $-0.58(0.25)$ & 0.68 & 0.37 & 0.62 & 0.95 & 0.10 & 0001 \\
\hline $\begin{array}{l}\text { PB8: Turning } 360 \\
\text { degrees }\end{array}$ & $-0.59(0.25)$ & 0.97 & 1.14 & 0.48 & 0.74 & 0.11 & 001 \\
\hline $\begin{array}{l}\text { BBS11: Turning } 360 \\
\text { degrees }\end{array}$ & $-0.59(0.25)$ & 0.95 & 0.75 & 0.50 & 0.74 & 0.00 & 00001 \\
\hline PG7: Step width & $-0.65(0.26)$ & 0.84 & 0.57 & 0.54 & 0.85 & 0.10 & 01 \\
\hline PG5: Gait path & $-0.72(0.26)$ & 0.88 & 1.49 & 0.49 & 0.78 & 0.22 & 01 \\
\hline PG6: Trunk sway & $-0.72(0.26)$ & 0.74 & 0.50 & 0.57 & 0.89 & 0.10 & 001 \\
\hline $\begin{array}{l}\text { BBS12: Stepping on } \\
\text { stool }\end{array}$ & $-1.02(0.28)$ & 0.88 & 1.29 & 0.46 & 0.75 & 0.10 & 00001 \\
\hline $\begin{array}{l}\text { PB7: Standing with } \\
\text { eyes closed }\end{array}$ & $-1.57(0.33)$ & 1.04 & 0.58 & 0.41 & 0.75 & 0.09 & 01 \\
\hline PB3: Attempts to arise & $-1.93(0.37)$ & 1.09 & 0.41 & 0.38 & 0.79 & 0.09 & 01 \\
\hline
\end{tabular}

Items are indicated by order number on the original scale; for e.g., BBS14 = item 14 on BBS. PTMEA = point-measure correlation coefficient.

${ }^{+}$Item difficulty measures in log-odds units.

* Rating scales can be interpreted as follows: for BBS14, 00112 indicates that the first and second original categories were collapsed to '0', the third and fourth categories were collapsed to ' 1 ', and the fifth category was unchanged, resulting in a 3-point scale. 
Table 3

Known-Groups Validity: Ability of 10-Item CAT and Individual Balance Measures to Discriminate between Fallers and Non-Fallers

\begin{tabular}{lcccc}
\hline Subgroups & 10-Item CAT & BBS & POMA & DGI \\
\hline Fallers $(\mathrm{n}=57)$ & $48.8(1.3)$ & $50.9(0.6)$ & $26.5(0.3)$ & $21.1(0.4)$ \\
Non-fallers $(\mathrm{n}=130)$ & $53.0(1.0)$ & $52.0(0.5)$ & $26.7(0.3)$ & $22.1(0.3)$ \\
$p$-value & 0.0068 & 0.13 & 0.57 & 0.048 \\
\hline
\end{tabular}

All scores reflect means (S.E.s) adjusted for study sub-sample in analysis of covariance. A $0.0125(0.05 / 4)$ level was used for significance testing across the four comparisons. 
Table 4

Predictive Validity: Multiple Linear Regression Results Comparing Ability of Baseline 10-Item CAT and Individual Balance Scores to Predict 16-Week LLFDI-Function Scores

\begin{tabular}{lccccc}
\hline Primary Predictor & Slope (S.E.) & $\boldsymbol{p}$-value & Standardized Slope & Type II Partial R & Model R$^{2}$ \\
\hline 10-Item CAT & $0.56(0.1)$ & $<0.0001$ & 0.46 & 0.15 & 0.46 \\
BBS & $0.90(0.2)$ & $<0.0001$ & 0.36 & 0.10 & 0.40 \\
POMA & $1.10(0.3)$ & 0.0018 & 0.28 & 0.06 & 0.37 \\
DGI & $1.30(0.3)$ & 0.0001 & 0.34 & 0.09 & 0.39 \\
\hline
\end{tabular}

Predictive validity testing was conducted in a sub-sample of 115 subjects with LLFDI-Function scores from the 16-week randomized controlled trial dataset. All four models controlled for age, gender, body mass index, trial intervention group, and number of medical diagnoses. Larger standardized slopes and Type II Partial $\mathrm{R}^{2}$ values indicate stronger prediction. 